\title{
Nuclear and Nucleon Matrix Elements
}

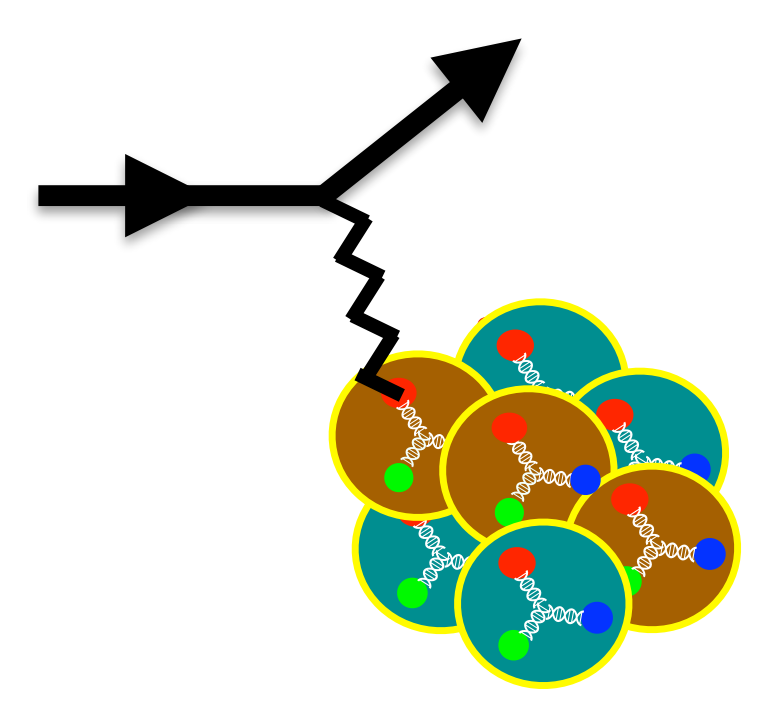

Michael Wagman

USQCD All Hands Meeting

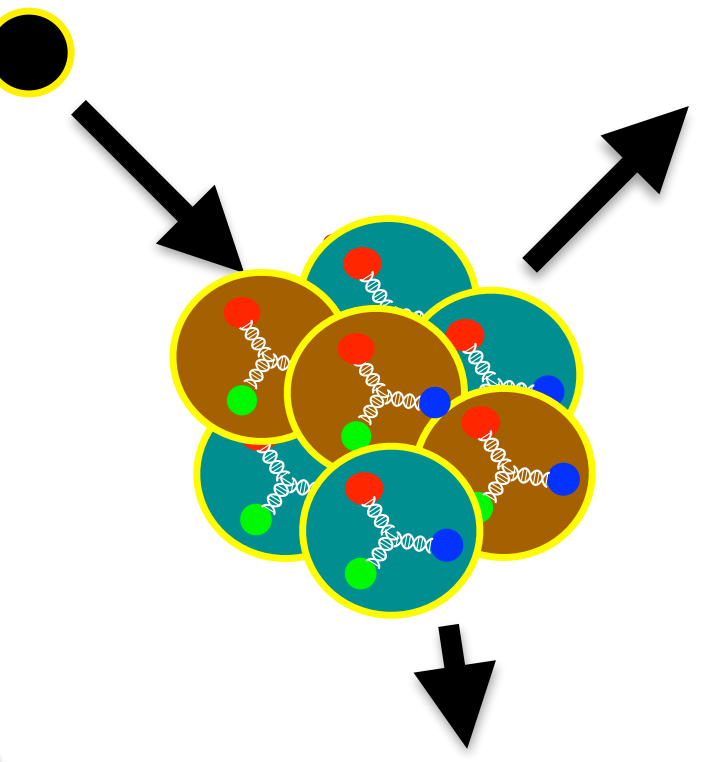

May 1, 2021

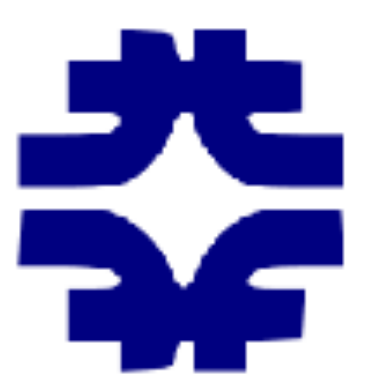

Fermilab

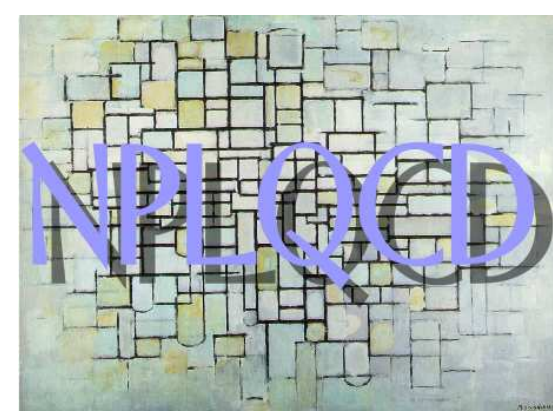




\section{Many-quark bound-state structure}

LQCD calculations of nucleon matrix elements are revealing fundamental aspects of hadron structure

See talks this afternoon, this talk will focus on nuclei in particular

The partonic structure of nuclei is noticeably different from the nucleon

Aubert et al (EMC), Phys. Lett. 123B (1983)

The emergence of the EMC effect and it's analogs from QCD is not yet understood
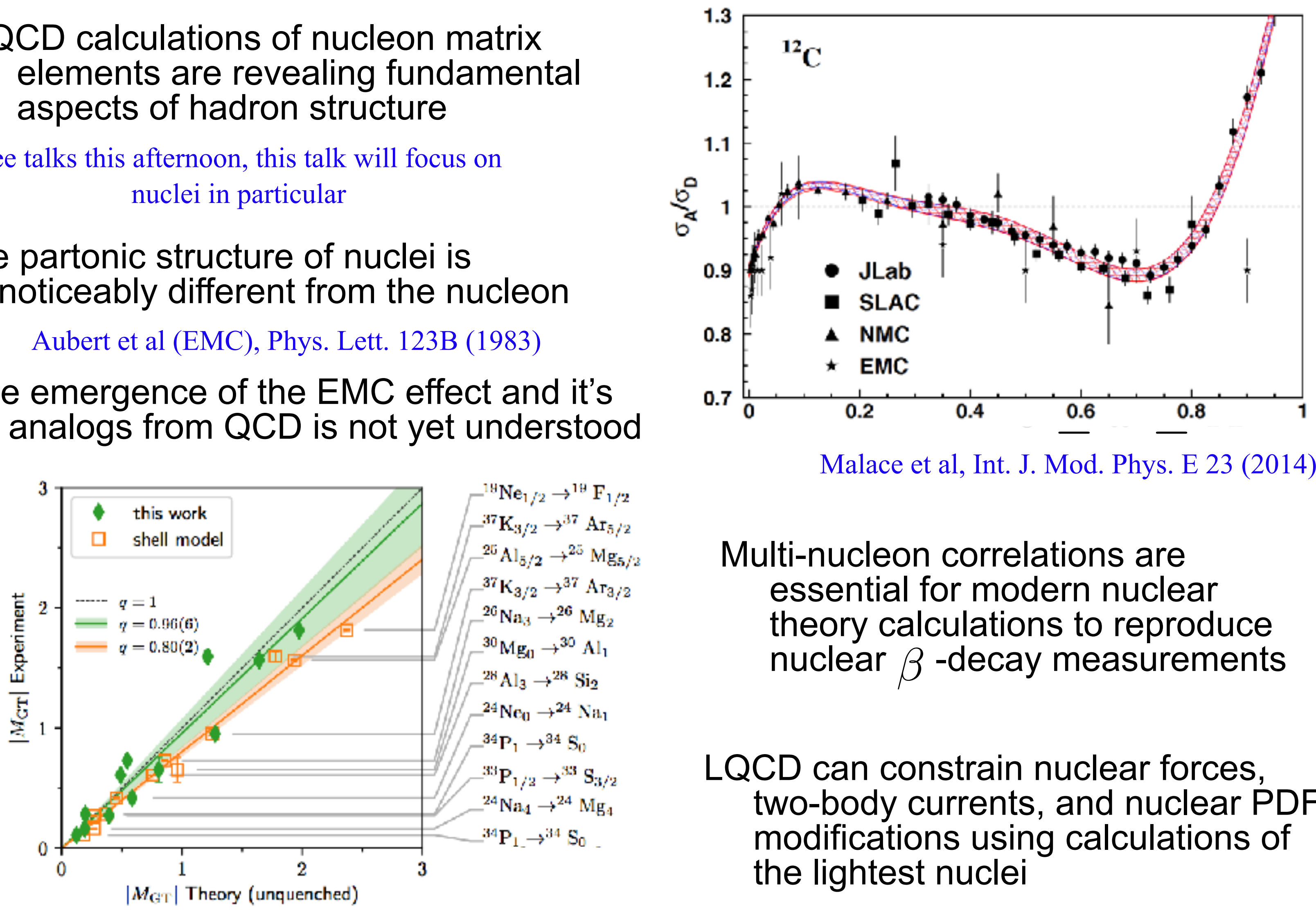

Multi-nucleon correlations are essential for modern nuclear theory calculations to reproduce nuclear $\beta$-decay measurements

LQCD can constrain nuclear forces, two-body currents, and nuclear PDF modifications using calculations of the lightest nuclei

Gysbers et al, Nature Phys. 15 (2019) 


\section{New physics and nuclei}

Nuclei are abundant and useful experimental targets

Converting between nuclear- and nucleon-level cross-sections requires

- Nuclear models (error bar ?s)

- Direct LQCD calculations (impractical)

- LQCD informed EFT + modeling

\section{Xenon1T constraint on Dark Matter-nucleus}

Next-generation accelerator neutrino experiments require

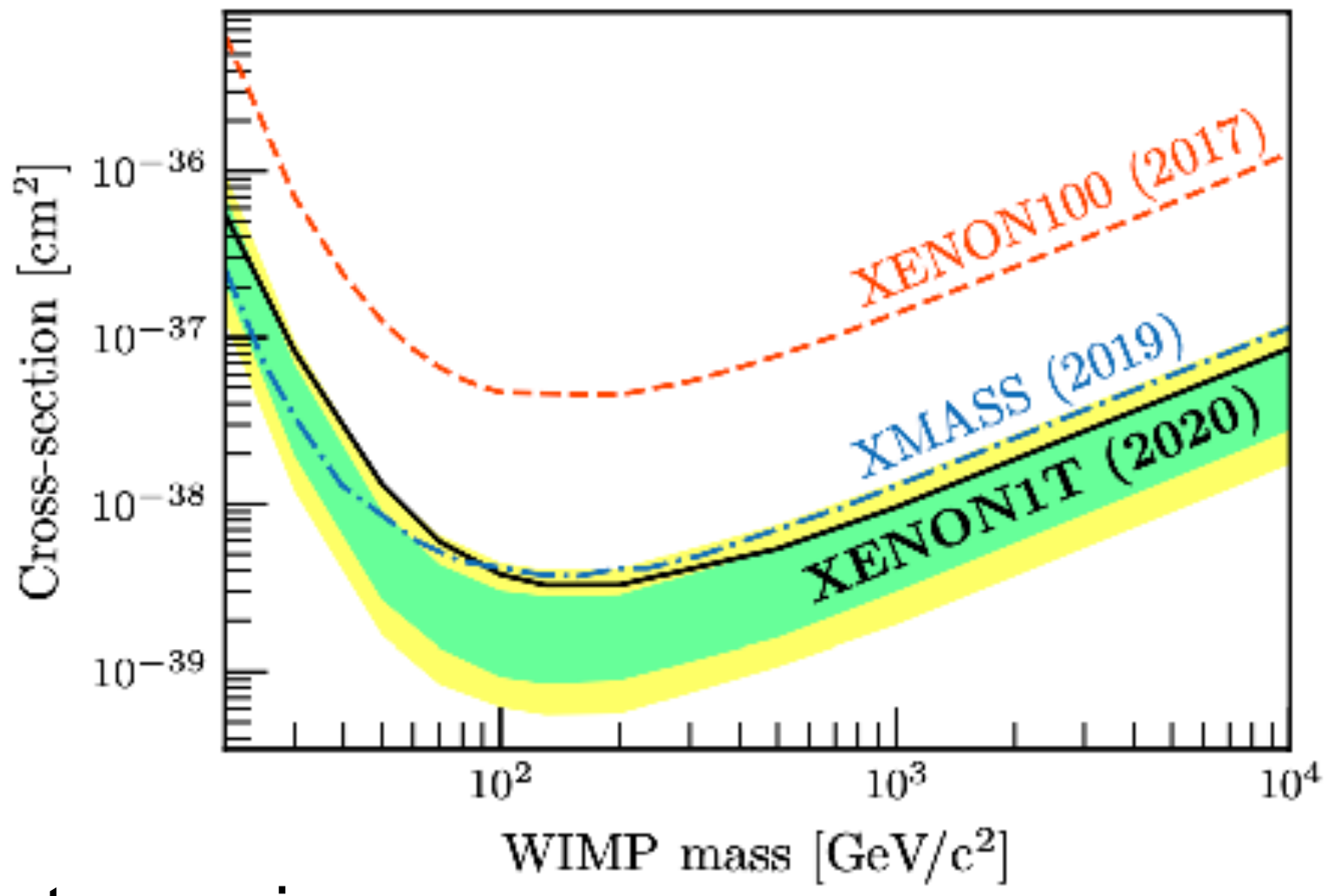
few-percent level control of nuclear cross-sections

Standard Model predictions with controlled uncertainties essential

DUNE

\section{Santord}

Underground

Research

Facility

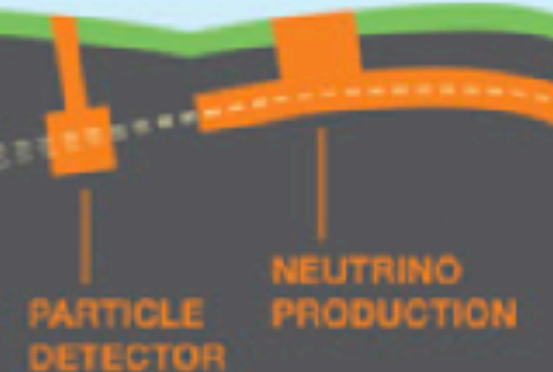

Fermilab PARTICLE 


\section{Nuclear matrix elements from LQCD}

Electroweak nuclear matrix elements and nuclear PDF moments extracted from $3 p t$ / 2pt function ratios, "just" need (often multi-local) nuclear interpolating operators

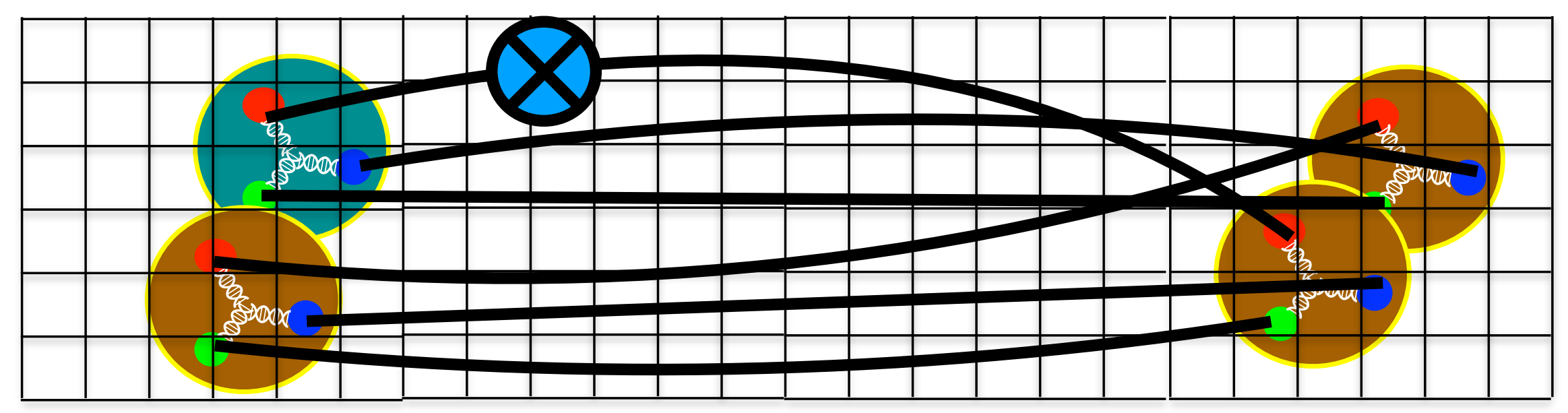

Fixed-order background field method simplifies three-point function contractions

Lattice methods, EFT matching strategies, and early nuclear ME results reviewed:

Davoudi et al, Phys.Rept. 900 (2021)

New results this year with $m_{\pi} \sim 450 \mathrm{MeV}$ and $m_{\pi} \sim 800 \mathrm{MeV}$

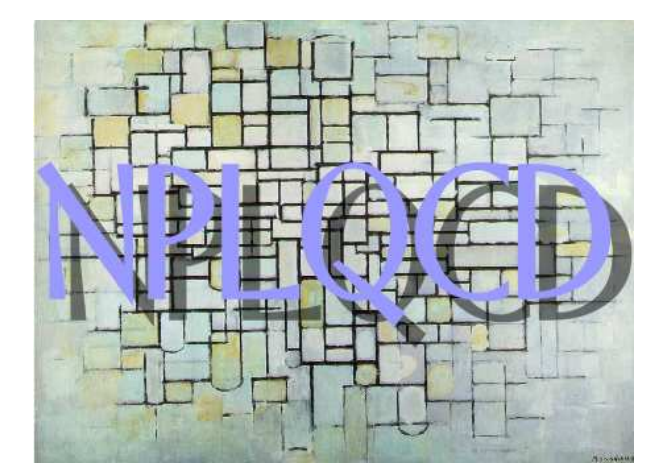

Detmold et al [NPLQCD]

arXiv:2009.05522

(accepted to PRL, in prep)

Illa et al [NPLQCD] PRD 103 (2021) Parreño et al [NPLQCD] PRD 103 (2021)

arXiv:2009.12357

arXiv:2102.03805 


\section{Nuclear matrix elements from LQCD}

Electromagnetic structure and $n p \rightarrow d \gamma$

Beane et al [NPLQCD] PRL 113 (2014)

Beane et al [NPLQCD] PRL 115 (2015)

Detmold et al [NPLQCD] PRL 116 (2016)

Proton-proton fusion and $2 \nu \beta \beta$ computed using fixed-order background field method

Savage et al [NPLQCD], PRL 119 (2017)

Shanahan et al [NPLQCD], PRL 119 (2017)

Tiburzi et al [NPLQCD], PRD 96 (2017)

Gluonic structure of light nuclei

Winter et al [NPLQCD], PRD 96 (2017)

Scalar, axial, and tensor matrix elements

Chang et al [NPLQCD], PRL 120 (2018)

$$
m_{\pi} \sim 800 \mathrm{MeV}
$$

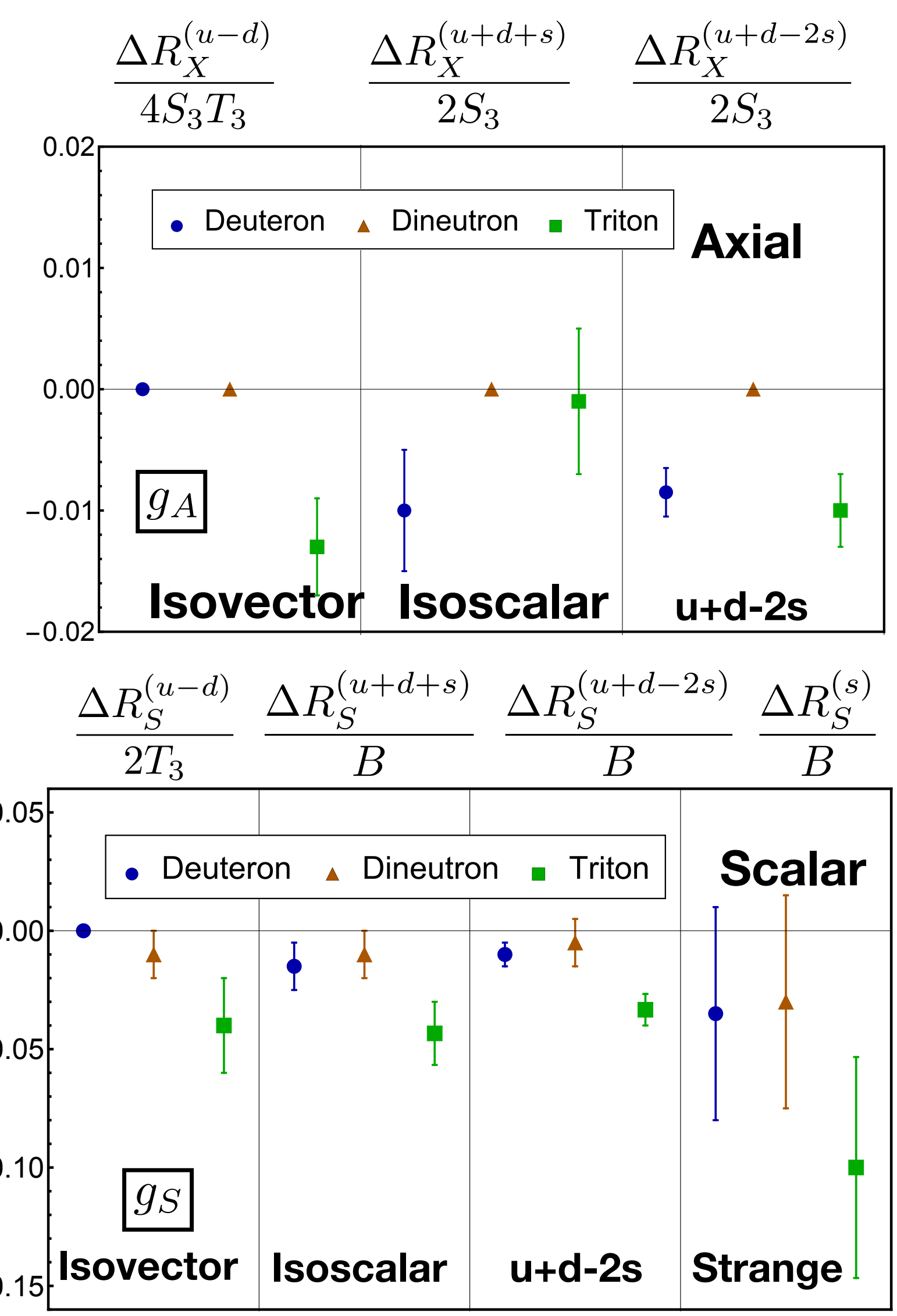




\section{Momentum fractions of light nuclei}

Momentum fraction carried by any quark flavor (or gluons) calculable from local matrix elements in generic hadrons

$$
\langle x\rangle_{h}^{i}=\int_{-1}^{1} d x x q_{h}^{i}(x)=C\left(M_{h}, \vec{p}, J\right) \sum_{\lambda=-J}^{J}\left\langle h, \vec{p}, \lambda\left|T_{\mu \nu}^{i}\right| h, \vec{p}, \lambda\right\rangle
$$

First calculation of isovector quark momentum fractions of light nuclei performed

Detmold et al [NPLQCD] PRL.xx $\quad$ arXiv:2009.05522

Although systematic uncertainties are not fully controlled (one lattice spacing, volume, quark mass, ...) demonstrates potential for LQCD to usefully constrain nuclear PDFs
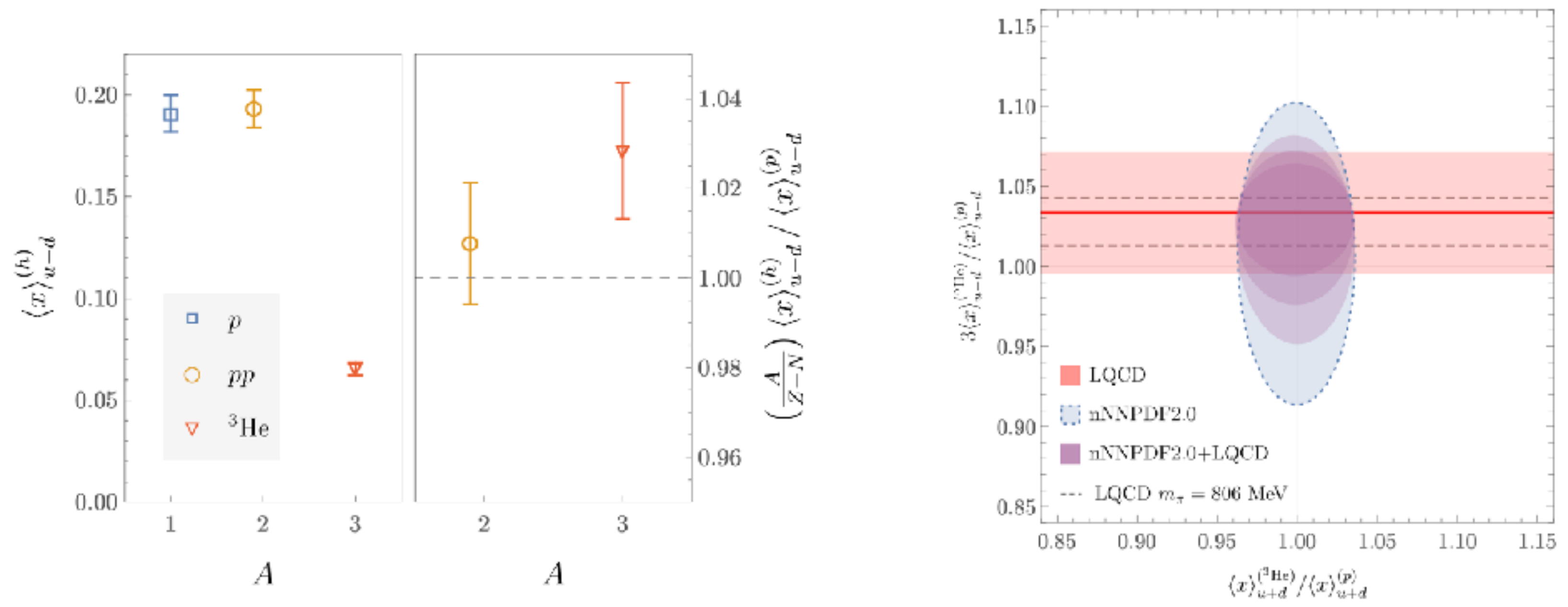


\section{Matching to nuclear EFT}

New analysis of baryon-baryon correlation functions allows (hyper-)nuclear forces to be constrained using pionless EFT

Illa et al [NPLQCD] PRD 103 (2021)

Approximate symmetries including SU(6) spin-flavor observed, though less pronounced than at SU(3) symmetric quark masses

$$
\begin{aligned}
& \mathcal{L}_{B B}^{(0), S U(3)}=-c_{1} \operatorname{Tr}\left(B_{i}^{\dagger} B_{i} B_{j}^{\dagger} B_{j}\right)-c_{2} \operatorname{Tr}\left(B_{i}^{\dagger} B_{j} B_{j}^{\dagger} B_{i}\right)-\sigma_{8} \operatorname{Tr}\left(B_{i}^{\dagger} B_{j}^{\dagger} B_{i} B_{j}\right) \\
& -c_{4} \operatorname{Tr}\left(B_{i}^{\dagger} B_{j}^{\dagger} B_{j} B_{i}\right)-c_{5} \operatorname{Tr}\left(B_{i}^{\dagger} B_{i}\right) \operatorname{Tr}\left(B_{j}^{\dagger} B_{j}\right)-c_{i j} \operatorname{Tr}\left(B_{i}^{\dagger} B_{j}\right) \operatorname{Tr}\left(B_{j}^{\dagger} B_{i}\right), \\
& \mathcal{L}_{B B}^{(2), S J(3)}=-\tilde{c}_{1} \operatorname{Tr}\left(B_{i}^{\dagger} \nabla^{2} B_{i} B_{j}^{\dagger} B_{j}+\text { h.c. }\right)-\tilde{c}_{2} \operatorname{Tr}\left(B_{i}^{\dagger} \nabla^{2} B_{j} B_{j}^{\dagger} B_{i}+\text { h.c. }\right) \\
& -\tilde{c}_{3} \operatorname{Tr}\left(B_{i}^{\dagger} B_{j}^{\dagger} \nabla^{2} B_{i} B_{j}+\text { h.c. }\right)-\tilde{c}_{4} \operatorname{Tr}\left(B_{i}^{\dagger} B_{j}^{\dagger} \nabla^{2} B_{j} B_{i}+\text { h.c. }\right) \\
& -\tilde{c}_{\delta}\left[\operatorname{Tr}\left(B_{i}^{\mid} \nabla^{2} B_{i}\right) \operatorname{Tr}\left(B_{j}^{\dagger} B_{j}\right)+\text { h.c. }\right]-\tilde{c}_{6}\left[\operatorname{Tr}\left(B_{i}^{\dagger} \nabla^{2} B_{j}\right) \operatorname{Tr}\left(B_{j}^{\dagger} B_{i}\right)+\right.\text { h.c.] }
\end{aligned}
$$

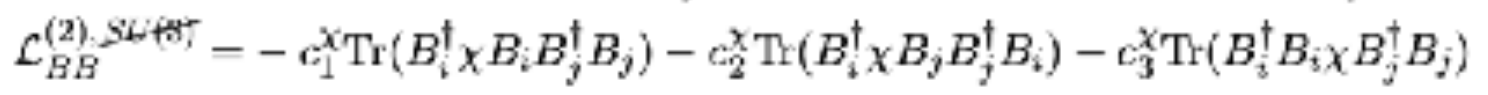

$$
\begin{aligned}
& -c_{4}^{\chi} \operatorname{Tr}\left(B_{i}^{\dagger} B_{j} \chi B_{j}^{\dagger} B_{i}\right)-c_{5}^{\chi} \operatorname{Tr}\left(B_{i}^{\dagger} \chi B_{j}^{\dagger} B_{i} B_{j}+\text { h.c. }\right)-c_{6}^{\chi} \operatorname{Tr}\left(B_{i}^{\dagger} \chi B_{j}^{\dagger} B_{j} B_{i}+\text { h.c. }\right) \\
& -c_{7}^{\chi} \operatorname{Tr}\left(B_{i}^{\dagger} B_{j}^{\dagger} \chi B_{i} B_{j}\right)-c_{8}^{\chi} \operatorname{Tr}\left(B_{i}^{\dagger} B_{j}^{\dagger} \chi B_{j} B_{i}\right)-c_{9}^{\chi} \operatorname{Tr}\left(B_{i}^{\dagger} B_{j}^{\dagger} B_{i} B_{j} \chi\right) \\
& -c_{10}^{\chi} \operatorname{Tr}\left(B_{i}^{\dagger} B_{j}^{\dagger} B_{j} B_{i} \chi\right)-c_{11}^{\chi} \operatorname{Tr}\left(B_{i}^{\dagger} \chi B_{i}\right) \operatorname{Tr}\left(B_{j}^{\dagger} B_{j}\right)-c_{12}^{\chi} \operatorname{Tr}\left(B_{i}^{\dagger} \chi B_{j}\right) \operatorname{Tr}\left(B_{j}^{\dagger} B_{i}\right) \text {, } \\
& \mathcal{L}_{B B}^{(0), S U(6)}=-a\left(\Psi_{\mu \nu \rho}^{\dagger} \Psi^{\mu \nu \rho}\right)^{2}-b \Psi_{\mu \nu \sigma}^{\dagger} \Psi^{\mu \nu \tau} \Psi_{\rho \delta \tau}^{\dagger} \Psi^{\rho \delta \sigma}
\end{aligned}
$$

Pionless EFT can also be used as a tool to extrapolate lattice QCD results to infinite-volume

Stochastic variational approach to infinitevolume extrapolation of energies

Barnea et al, PRL 114 (2015)

Recently extended to infinite-volume extrapolation of nuclear matrix elements

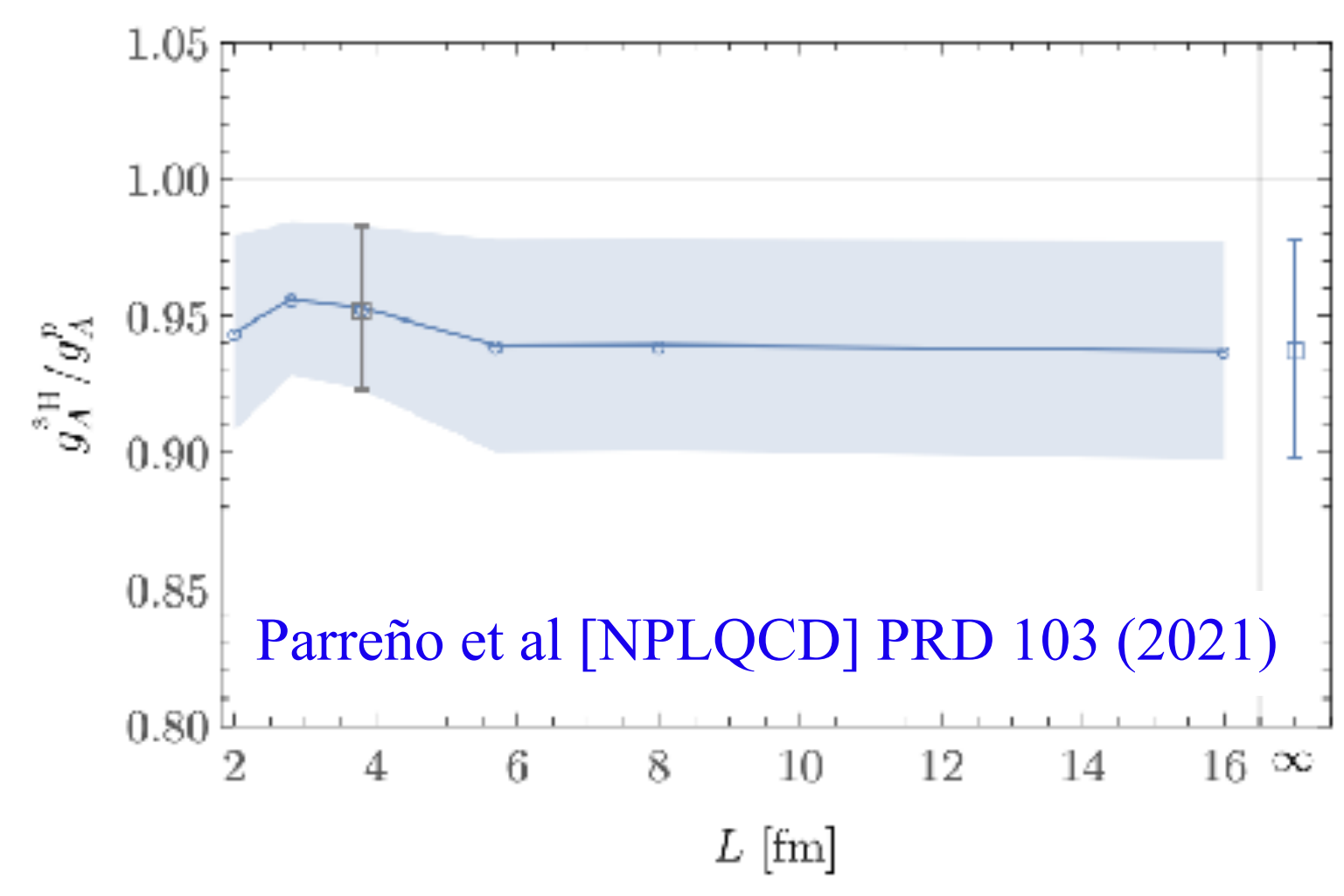

Detmold and Shanahan, PRD 103 (2021) 


\section{Triton $\beta$-decay}

Triton studied with one $m_{\pi} \sim 450 \mathrm{MeV}$ ensemble, 3 lattice volumes

Robust fitting methodology used to study excited-state effects

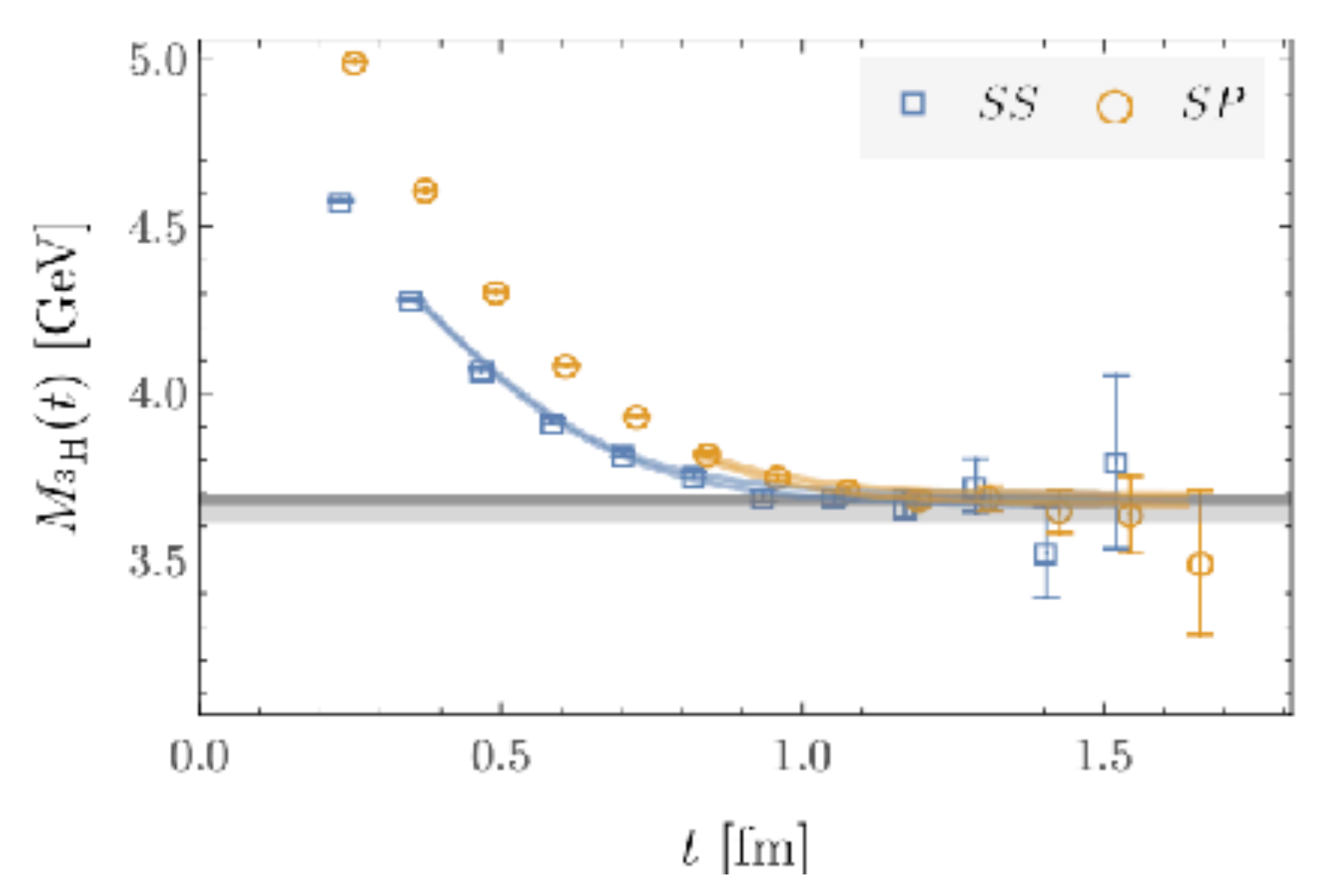

Ground-state energy consistent with a binding energy intermediate between nature and $m_{\pi} \sim 800 \mathrm{MeV}$

Gamow-Teller matrix element governing triton $\beta$-decay rate computed

$$
\begin{aligned}
g_{A}\left({ }^{3} \mathrm{H}\right) & =\left|\left\langle{ }^{3} \mathrm{He}\left|A_{z}^{+}\right|{ }^{3} \mathrm{H}\right\rangle\right| \\
& =\left|\left\langle{ }^{3} \mathrm{H}\left|A_{z}^{3}\right|{ }^{3} \mathrm{H}\right\rangle\right|
\end{aligned}
$$

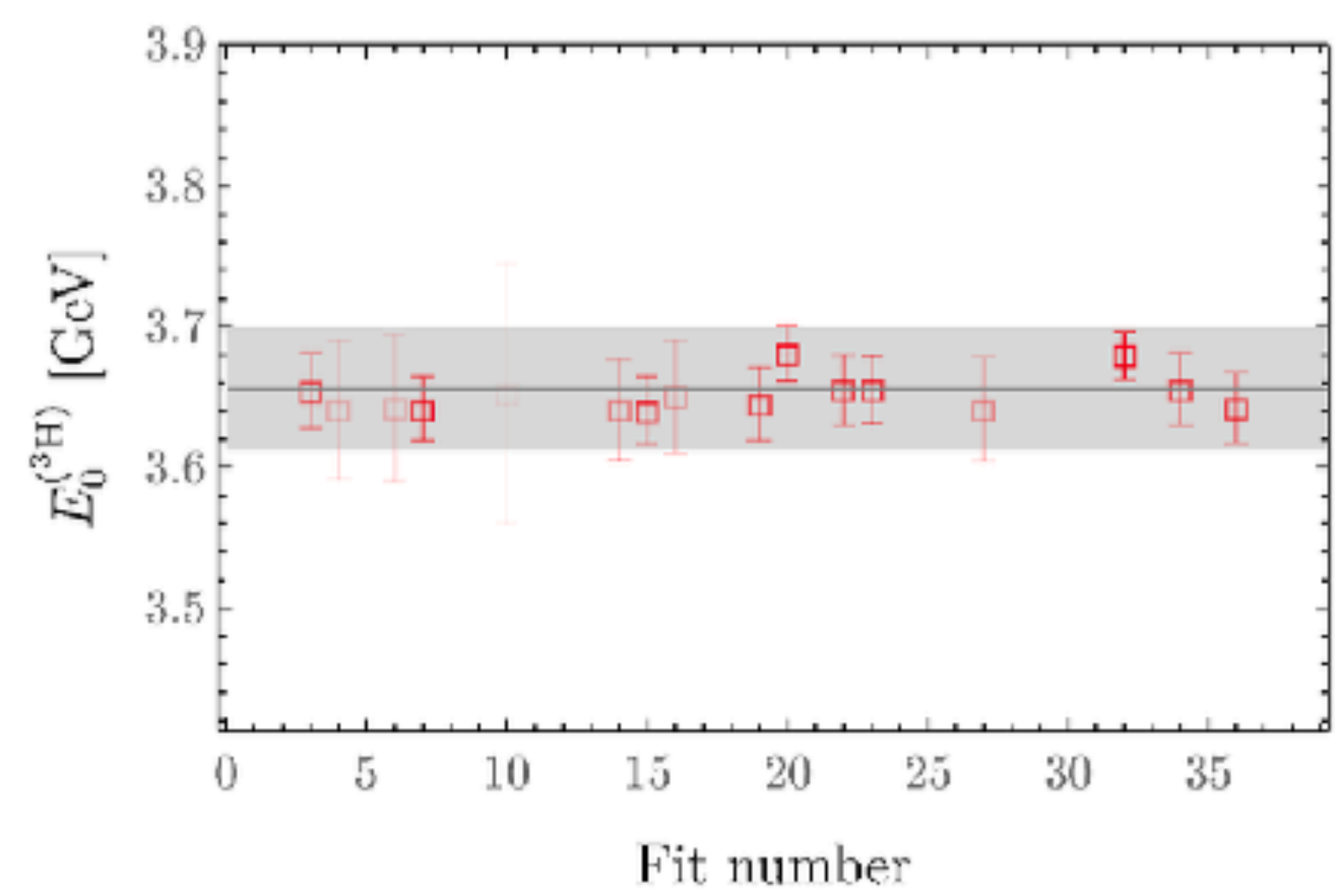

Parreño et al [NPLQCD] PRD 103 (2021) 


\section{Triton $\beta$-decay}

Triton $\beta$ - decay results with $m_{\pi} \sim 450 \mathrm{MeV}$ permit first extrapolations to physical point

Several systematic uncertainties remain, but encouraging agreement with experiment seen
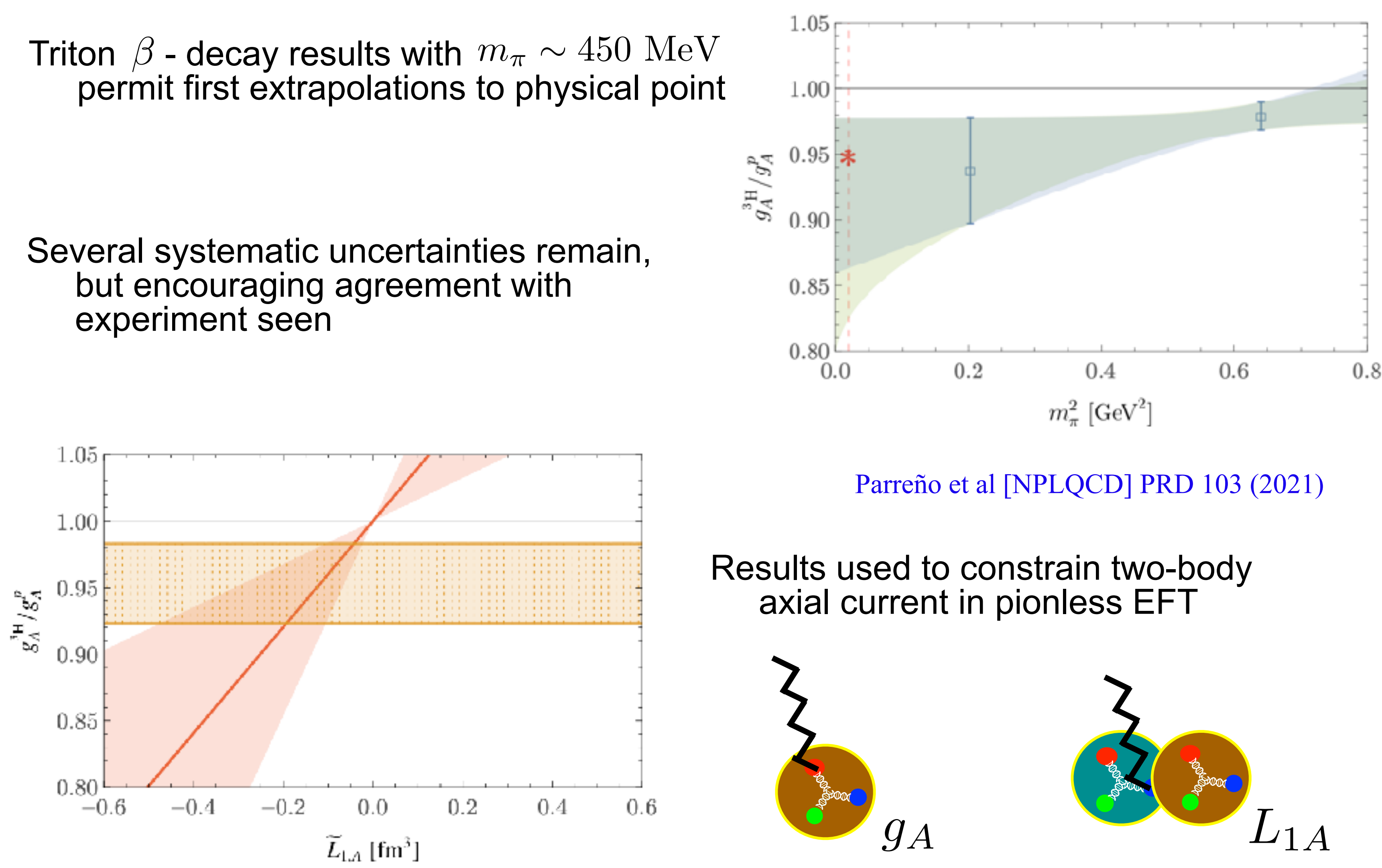

Parreño et al [NPLQCD] PRD 103 (2021)

Results used to constrain two-body axial current in pionless EFT
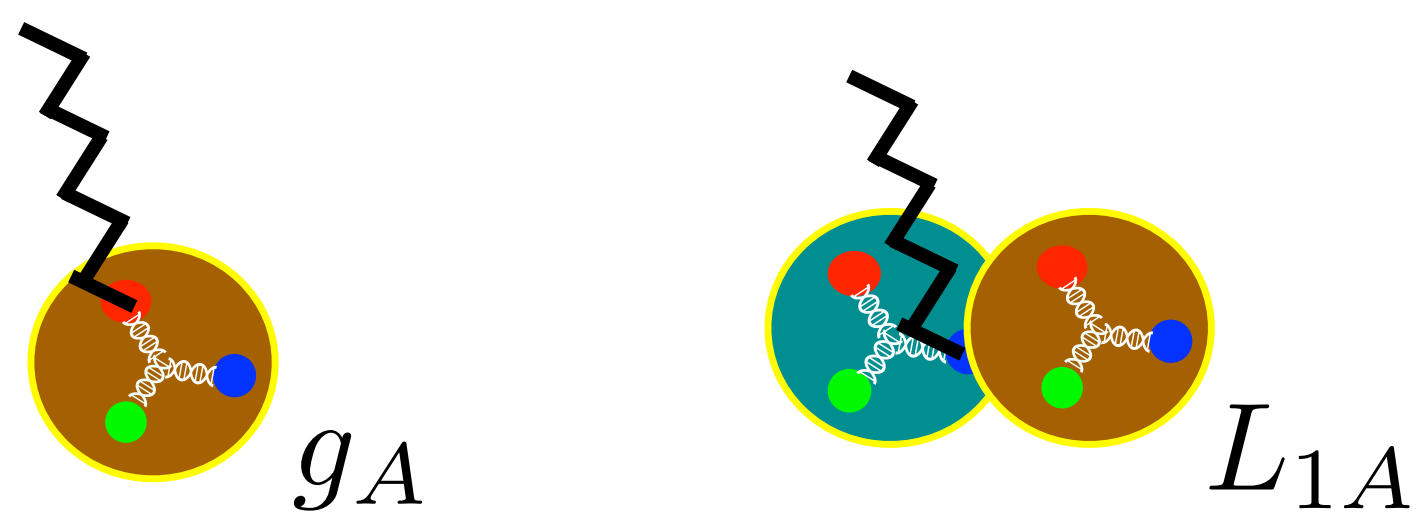


\section{Approaching the physical point}

Very high statistics required

Improved Wilson fermions used for efficiency and simplicity of contractions / spin algebra

QUDA multigrid with many propagator sources per configuration

$$
\begin{array}{ll} 
& \multicolumn{2}{c}{e^{3\left(2 M_{N}-3 m_{\pi}\right)(1 \mathrm{fm})}} \\
m_{\pi} \sim 800 \mathrm{MeV}: & 10^{5} \\
m_{\pi} \sim 450 \mathrm{MeV}: & 10^{7} \\
m_{\pi} \sim 140 \mathrm{MeV}: & 10^{9}
\end{array}
$$

Clark et al, Proceedings SC '16 (2016)

QDP / JIT used to put all parts of propagator calculations on GPU

Winter, Clark, Edwards, Jóo, Proceedings IPDPS ’14 (2014)

Sparsened quark propagators and baryon blocks allow manageable storage 


\section{Systematic uncertainties}

Several systematic uncertainties remain to be quantified in detail

- Heavier than physical quark masses only

- One lattice spacing

- Excited-state effects 


\section{Systematic uncertainties}

Several systematic uncertainties remain to be quantified in detail

- Heavier than physical quark masses only

- One lattice spacing

- Excited-state effects

Gap between ground and two-nucleon finite-volume "scattering" states becomes small for large volumes, ground-state dominance relies on overlap factors

$$
Z_{0} e^{-E_{0} t}\left(1+\frac{Z_{1}}{Z_{0}} e^{-\delta t}+\ldots\right) \quad \delta \sim \frac{4 \pi^{2}}{M L^{2}}
$$

For non-positive-definite correlation functions, cancellations between the ground and excited-state could in principle conspire to form a "false plateau"

See e.g. Iritani et al, JHEP 10 (2016)

First studies using positive-definite correlation functions (enabled by distillation / stochastic LapH) give results in tension with previous studies 


\section{Thinking positive}

Correlation-function matrices for an interpolator set including both local "hexaquark" and bilocal "dibaryon" operators generalize calculations performed to date
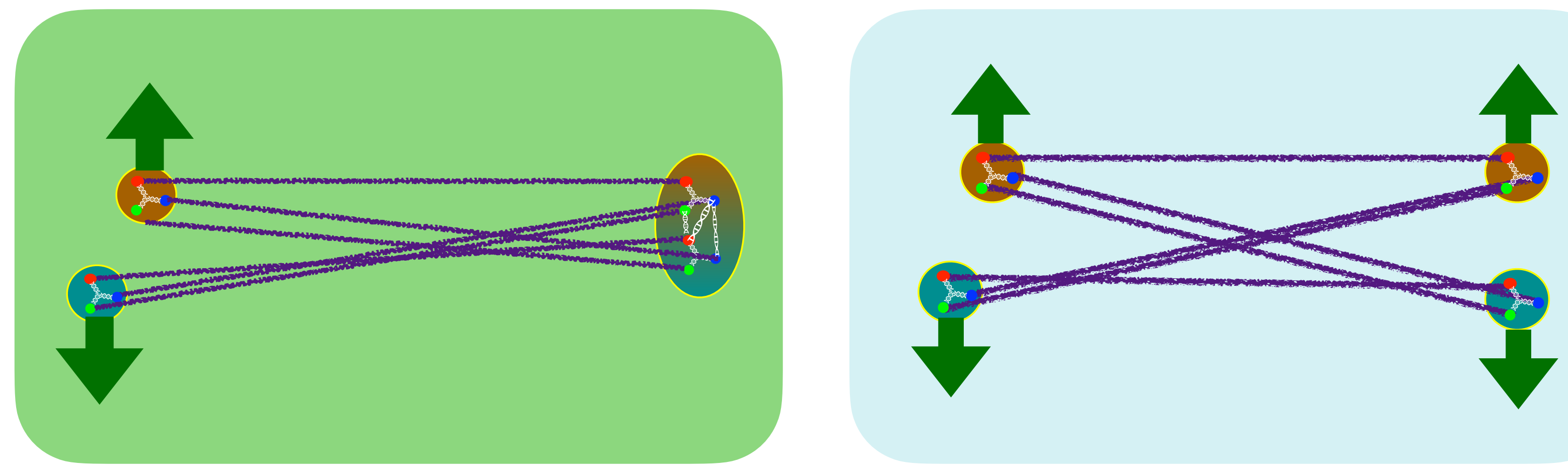

Also possible to include "quasi-local" operators with a variety of wavefunctions in an interpolating operator set, may increase overlap with loose bound states
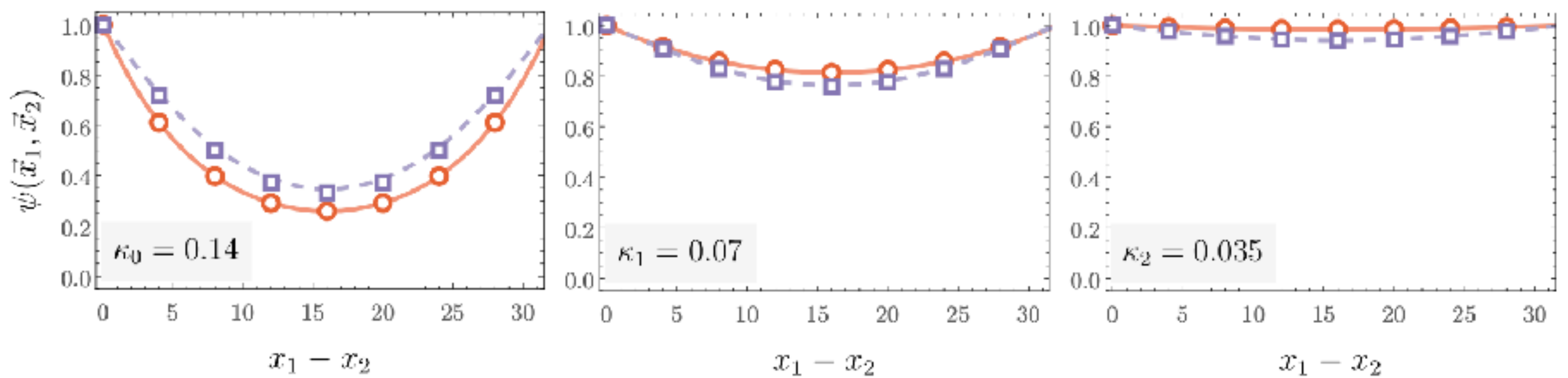


\section{Contractions - a piece of cake}
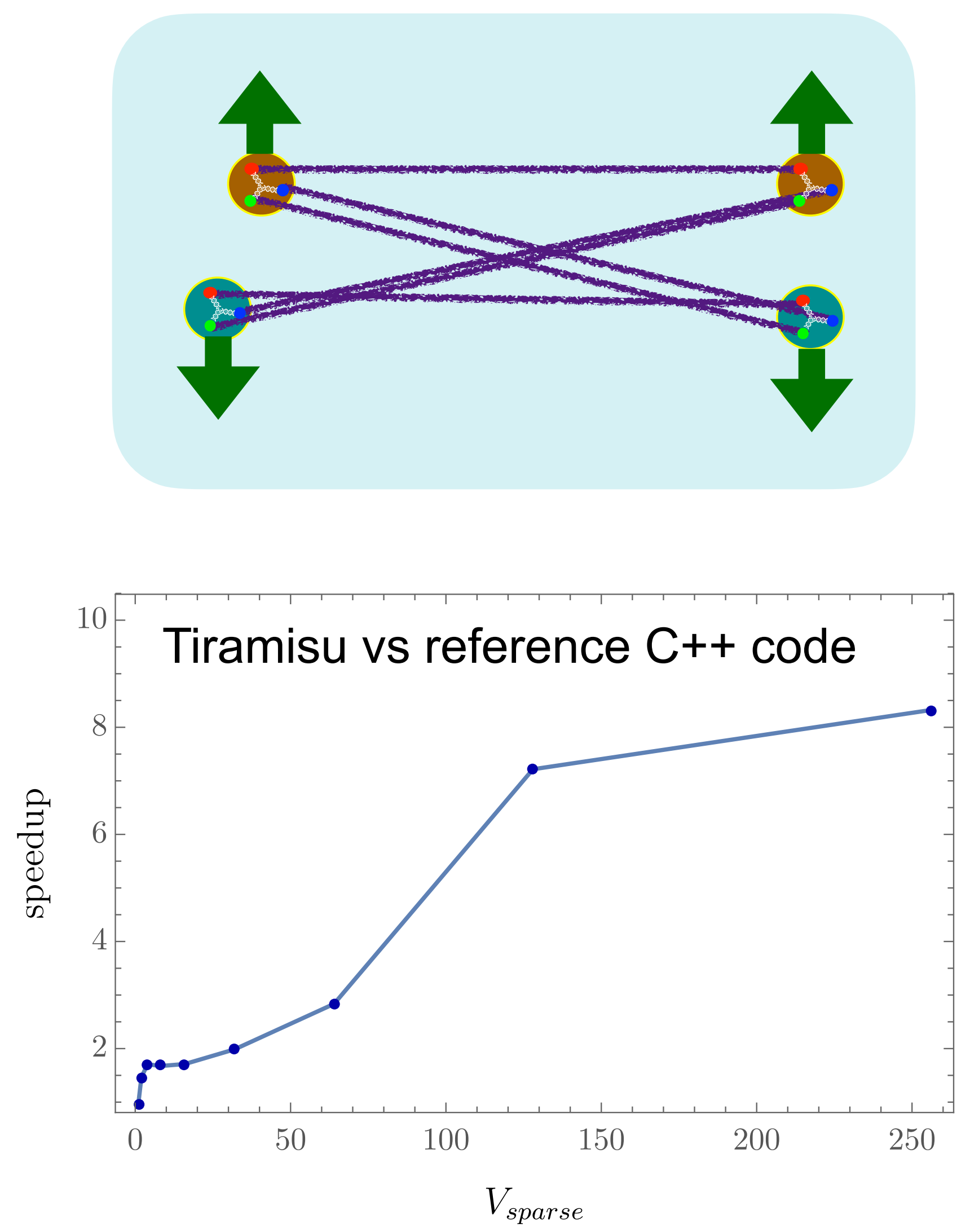

Loops over independent spatial locations

for each baryon are expensive

- Bilocal baryon blocks

(product of sums)

$$
V^{4} \rightarrow V^{3}
$$

- Sparsening

$$
V \rightarrow V_{\text {sparse }}
$$

- Tiramisu - a C++ polyhedral compiler that excels at optimizing tensor contractions

Baghdadi et al, arXiv:2005.04091

https://github.com/Tiramisu-Compiler/tiramisu 


\section{Towards variational studies of nuclei}

New contraction codes validated

(including machine precision

symmetry tests) and CPU version

optimized

Promising preliminary results obtained by solving Generalized Eigenvalue

Problem (GEVP) with variational sets of two-nucleon interpolating operators

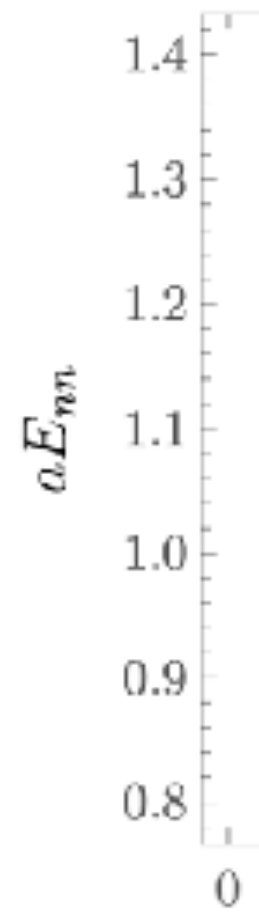

\begin{tabular}{ccccccc}
\hline Flavor States & $I$ & $J$ & $-S$ & $N_{w}: N_{d}: N_{\varepsilon}$ & Cost & Storage \\
\hline$n p$ & 0 & 1 & 0 & $3: 3: 0$ & 108 & 3 \\
$p p$ & 1 & 0 & 0 & $4: 2: 0$ & 48 & 1 \\
\hline$\Lambda p, \Sigma^{0} p, \Sigma^{+} n$ & $1 / 2$ & 0 & 1 & $3: 2: 1$ & 108 & 9 \\
$\Lambda p, \Sigma^{0} p, \Sigma^{+} n$ & $1 / 2$ & 1 & 1 & $3: 2: 1$ & 324 & 27 \\
$\Sigma^{+} p$ & $3 / 2$ & 0 & 1 & $4: 1: 1$ & 24 & 1 \\
$\Sigma^{+} p$ & $3 / 2$ & 1 & 1 & $4: 1: 1$ & 72 & 3 \\
\hline$\Xi^{-} p, \Xi^{0} n, \Lambda \Lambda, \Sigma^{+} \Sigma^{-}, \Sigma^{0} \Sigma^{0}$ & 0 & 0 & 2 & $2: 2: 2$ & 400 & 25 \\
$\Xi^{-} p, \Xi^{0} n$ & 0 & 1 & 2 & $2: 2: 2$ & 96 & 12 \\
$\Lambda \Sigma^{+}, \Xi^{0} p$ & 1 & 0 & 2 & $3: 1: 2$ & 48 & 4 \\
$\Lambda \Sigma^{+}, \Sigma^{0} \Sigma^{+}, \Xi^{0} p$ & 1 & 1 & 2 & $3: 1: 2$ & 324 & 27 \\
$\Sigma^{+} \Sigma^{+}$ & 2 & 0 & 2 & $4: 0: 2$ & 48 & 1 \\
$\Lambda \Xi^{0}, \Sigma^{0} \Xi^{0}, \Sigma^{+} \Xi$ & $1 / 2$ & 0 & 3 & $2: 1: 3$ & 108 & 9 \\
$\Lambda \Xi^{0}, \Sigma^{0} \Xi^{0}, \Sigma^{+} \Xi^{-}$ & $1 / 2$ & 1 & 3 & $2: 1: 3$ & 324 & 27 \\
$\Sigma^{+} \Xi^{0}$ & $3 / 2$ & 0 & 3 & $3: 0: 3$ & 36 & 1 \\
$\Sigma^{+} \Xi^{0}$ & $3 / 2$ & 1 & 3 & $3: 0: 3$ & 108 & 3 \\
\hline$\Xi^{0} \Xi^{-}$ & 0 & 1 & 4 & $1: 1: 4$ & 72 & 3 \\
$\Xi^{0} \Xi^{0}$ & 1 & 0 & 4 & $2: 0: 4$ & 48 & 1 \\
\hline & & & & Total & 2296 & 157
\end{tabular}

$$
m_{\pi} \sim 170 \mathrm{MeV}
$$$$
\text { 回 }
$$$$
\text { 曰田 }
$$$$
\text { 口回四 }
$$$$
\text { 回回品 }
$$$$
\text { 回 }
$$$$
\text { 回 }
$$

$$
t / a
$$

Next step: variational baryonbaryon scattering including coupled-channel systems relevant for $\mathrm{H}$-dibaryon and neutron star physics

LQCD calculations of nuclear matrix elements with fully controlled systematic uncertainties on the horizon 